\title{
UNIQUENESS OF JOSEPH IDEAL
}

\author{
Wee Teck Gan and Gordan Savin
}

\section{Introduction}

Let $\mathfrak{g}$ be a simple complex Lie algebra. By the PBW theorem, the universal enveloping algebra $U(\mathfrak{g})$ has a filtration $U_{n-1}(\mathfrak{g}) \subset U_{n}(\mathfrak{g})$ so that $U_{n}(\mathfrak{g}) / U_{n-1}(\mathfrak{g})$ is naturally isomorphic to the $n$-th symmetric power $S^{n}(\mathfrak{g})$ of $\mathfrak{g}$. For any twosided ideal $\mathcal{J}$ of $U(\mathfrak{g})$, we can associate an ideal $J=\operatorname{gr}(\mathcal{J})$ in the symmetric algebra $S(\mathfrak{g})=\oplus_{n=0}^{\infty} S^{n}(\mathfrak{g})$ defined by

$$
J=\oplus_{n=1}^{\infty} \mathcal{J}_{n} / \mathcal{J}_{n-1}
$$

where $\mathcal{J}_{n}=\mathcal{J} \cap U_{n}(\mathfrak{g})$. The zero set defined by $J$ in $\mathfrak{g} \cong \mathfrak{g}^{*}$ is the associated variety of $\mathcal{J}$ and will be denoted by $\operatorname{Ass}(\mathcal{J})$. If $\mathcal{J}$ is primitive, then $\operatorname{Ass}(\mathcal{J})$ is contained in the nilpotent cone of $\mathfrak{g}$.

The Lie algebra $\mathfrak{g}$ has a unique minimal (non-trivial) nilpotent orbit $\mathbf{O}_{\min }$. If $\alpha$ is the highest root, and $\left(e_{\alpha}, h_{\alpha}, e_{-\alpha}\right)$ is the standard $s l(2)$-triple corresponding to $\alpha$, then $\mathbf{O}_{\min }$ is simply the adjoint orbit of $e_{\alpha}$.

In an important paper $[\mathrm{J}]$, Joseph constructed a completely prime 2-sided primitive ideal $\mathcal{J}_{0}$ whose associated variety is $\overline{\mathbf{O}}_{\text {min }}$. He also derived a number of properties of $\mathcal{J}_{0}$; for example he computed its infinitesimal character. We shall refer to $\mathcal{J}_{0}$ as the Joseph ideal. Joseph also proved that, when $\mathfrak{g}$ is not of type $\mathrm{A}, \mathcal{J}_{0}$ is the unique completely prime two-sided ideal whose associated variety is the closure of the minimal orbit. However, it was noticed by the second author $[\mathrm{S}]$ that there is a gap in Joseph's proof, namely in the proof of [J, Lemma 8.8] (see the remark at the end of $\S 2$ ). This is somewhat undesirable, given the subsequent importance of the Joseph ideal in representation theory, especially in the theory of minimal representations.

In this paper, we provide a simple proof of the uniqueness of the Joseph ideal. Our proof is different from the one envisioned in Joseph's original paper [J]. It is possible that the uniqueness can be deduced from some other results in the literature. For example, Braverman and Joseph have informed us that it should be a consequence of the results of their paper [BJ]. There, they considered a one-parameter (non-commutative) deformation of the coordinate ring of $\overline{\mathbf{O}}_{\text {min }}$ and showed that there is a unique deformation which gives rise to the Joseph ideal. This gives an alternative construction of the Joseph ideal. However, to show uniqueness, one would need to show that given a completely prime ideal

Received February 18, 2004. 
$\mathcal{J}$ with associated variety $\overline{\mathbf{O}}_{\text {min }}, U(\mathfrak{g}) / \mathcal{J}$ belongs to the one-parameter family of deformations considered in [BJ]. This does not seem to be addressed in [BJ], but could perhaps be shown using Proposition 2.1 below.

\section{Joseph Ideal}

We assume henceforth that $\mathfrak{g}$ is not of type $A$ and begin by describing some results of Kostant and Garfinkle [G].

Let $J_{0}$ be the prime ideal in the symmetric algebra $S(\mathfrak{g})$ corresponding to the closure of the minimal nilpotent orbit in $\mathfrak{g}^{*}$. Kostant has shown that, as a $\mathfrak{g}$-module,

$$
S(\mathfrak{g}) / J_{0}=\sum_{m=0}^{\infty} V(m \alpha),
$$

and that $J_{0}$ is generated by a $\mathfrak{g}$-submodule $V(0)+W$ in $S^{2}(\mathfrak{g})$, such that

$$
S^{2}(\mathfrak{g})=V(2 \alpha) \oplus V(0) \oplus W .
$$

The structure of $W$ was determined by Garfinkle as follows.

Assume first that $\mathfrak{g}$ is not of type $C_{n}$. Let $\mathfrak{m}$ be the centralizer of $h_{\alpha}$. It is a reductive subalgebra whose simple roots are precisely the simple roots of $\mathfrak{g}$ perpendicular to $\alpha$. Write $[\mathfrak{m}, \mathfrak{m}]=\oplus \mathfrak{m}_{i}$ as a direct sum of simple summands. Let $\alpha_{i}$ be the highest root of the summand $\mathfrak{m}_{i}$. Then, if $\mathfrak{g}$ is not of type $C_{n}$

$$
W=\oplus_{i} V\left(\alpha+\alpha_{i}\right) \text {. }
$$

Note that each irreducible $\mathfrak{g}$-module $V\left(\alpha+\alpha_{i}\right)$ can be considered, in a canonical fashion, as a submodule of $U_{2}(\mathfrak{g})$, as it appears with multiplicity one there.

On the other hand, if $\mathfrak{g}$ is of type $C_{n}$, then $[\mathfrak{m}, \mathfrak{m}]$ is simple of type $C_{n-1}$, so that there is a unique $\alpha_{1}$ (the highest root of $[\mathfrak{m}, \mathfrak{m}]$ ). In this case,

$$
W=V\left(\alpha+\alpha_{1}\right) \oplus V\left(\frac{1}{2}\left(\alpha+\alpha_{1}\right)\right) .
$$

Now we have a crucial proposition:

Proposition 2.1. Let $\mathcal{J}$ be a completely prime ideal in $U(\mathfrak{g})$ such that $A s s(\mathcal{J})=$ $\overline{\mathbf{O}}_{\text {min }}$. Then $\mathcal{J}$ contains $W$ and $\Omega-c$, where $\Omega$ is the Caismir element of $U(\mathfrak{g})$ and $c$ is a constant.

Proof. If $A s s(\mathcal{J})=\overline{\mathbf{O}}_{\text {min }}$, then $J$ contains a power of $J_{0}$. Since $J_{0}^{k}$ is generated by the symmetric power $S^{k}(V(0)+W)$ over $S(\mathfrak{g})$, it follows that the $\mathfrak{g}$-types of $J_{0}^{k} / J_{0}^{k+1}$ are contained in

$$
S^{k}(V(0)+W) \otimes\left(\oplus_{m=0}^{\infty} V(m \alpha)\right)
$$

Thus, the highest weights of $\mathfrak{g}$-types in $S(\mathfrak{g}) / J$ are located on finitely many lines parallel to $\alpha$. In particular, for all sufficiently large integers $n, V\left(n \alpha+n \alpha_{i}\right)$ does not appear as a submodule of $S(\mathfrak{g}) / J$. Since $S(\mathfrak{g}) / J$ is isomorphic to $U(\mathfrak{g}) / \mathcal{J}$ as 
a $\mathfrak{g}$-module, $V\left(n \alpha+n \alpha_{i}\right)$ does not appear as a submodule of $U(\mathfrak{g}) / \mathcal{J}$ for large $n$.

Thus, if $a$ is a highest weight vector of an irreducible constituent of $W \subset$ $U_{2}(\mathfrak{g})$, then $a^{n}=0$ (modulo $\mathcal{J}$ ) for sufficiently large $n$. Since $\mathcal{J}$ is completely prime, a must be in $\mathcal{J}$, so that the first part holds.

Similarly, since the multiplicity of the trivial $\mathfrak{g}$-module in $U(\mathfrak{g}) / \mathcal{J}$ is finite, $P(\Omega)$ is in $\mathcal{J}$ for some polynomial $P$. Since $P$ can be factored into linear factors, and $\mathcal{J}$ is completely prime, it follows that $P$ can be taken to be of degree one.

In $[J]$, Joseph constructed a primitive ideal $\mathcal{J}$ which is completely prime with $A s s(\mathcal{J})=\overline{\mathbf{O}}_{\text {min }}$, and computed its infinitesimal character. Using Proposition 2.1 , we can now give an alternative description of $\mathcal{J}$.

Proposition 2.2. (Garfinkle [G]) The Joseph ideal $\mathcal{J}$ is equal to the ideal $\mathcal{J}_{0}$ generated by $W$ and $\Omega-c_{0}$, where $c_{0}$ is the eigenvalue of $\Omega$ for the infinitesimal character that Joseph obtained.

Proof. Proposition 2.1 implies that the Joseph ideal $\mathcal{J}$ contains $W$. Since $\Omega-c_{0}$ is in $\mathcal{J}$ as well, it follows that $\mathcal{J} \supseteq \mathcal{J}_{0}$ and, for their graded versions, $J \supseteq$ $J_{0}$. If $J_{0} \neq J$, then since $J_{0}$ is prime, the associated variety of $\mathcal{J}$ would be a proper invariant subvariety of $\overline{\mathbf{O}}_{\text {min }}$. It follows that $A s s(\mathcal{J})=\{0\}$. This is a contradiction, and thus $J=J_{0}$, which implies that $\mathcal{J}=\mathcal{J}_{0}$. The proposition is proved.

Remarks: Garfinkle's proof of this proposition is based on the uniqueness of the Joseph ideal. The proof of uniqueness, however, has a gap in [J, Lemma 8.8]. More precisely, using the notations of [J], several lines before the end of the proof, there is an equation

$$
a^{n} u_{n}+\ldots u_{0}=0 .
$$

Since $a$ is in $J_{0}$, Joseph concluded that $u_{0}$ is in $J_{0}$. This is the mistake. Indeed, the above equation only holds modulo $J$. So one can only conclude that $u_{0}$ is in $J+J_{0}=U(\mathfrak{g})$, unless $J_{0} \supseteq J$, which is what Joseph wanted to prove with this argument.

\section{Uniqueness of $\mathcal{J}_{0}$.}

In this section we shall prove the uniqueness of $\mathcal{J}_{0}$ when $\mathfrak{g}$ is not of type A.

Theorem 3.1. Let $\mathfrak{g}$ be a simple complex Lie algebra which is not of type A. Let $\mathcal{J}$ be a completely prime two-sided ideal in $U(\mathfrak{g})$ such that its associated variety is $\overline{\mathbf{O}}_{\text {min }}$. Then $\mathcal{J}$ is the Joseph ideal $\mathcal{J}_{0}$.

Proof. By Proposition 2.1, $\mathcal{J}$ contains $W$ and $\Omega-c$ for some constant $c$. In view of Garfinkle's results, to show that $\mathcal{J}=\mathcal{J}_{0}$, we need to show that $c=c_{0}$, where $c_{0}$ is the eigenvalue of $\Omega$ for $\mathcal{J}_{0}$. 
Now the value of $c$ is determined by the intersection

$$
(\mathfrak{g}+\Omega \cdot \mathfrak{g}) \cap \mathcal{J} .
$$

Since $\mathcal{J}$ contains $W$, to show that $\mathcal{J}$ is equal to $\mathcal{J}_{0}$ (i.e. $c=c_{0}$ ), it suffices to show that

$$
\mathfrak{g} \cdot W \cap(\mathfrak{g}+\Omega \cdot \mathfrak{g}) \neq 0 .
$$

Indeed, this will show that $c$ is independent of $\mathcal{J}$. It will be more convenient to pass to the symmetric algebra $S(\mathfrak{g})$, where we need to prove that

$$
\mathfrak{g} \cdot W \supset \Omega \cdot \mathfrak{g} .
$$

To do so, we need to recall some results of Chevalley and Kostant $[\mathrm{K}]$ on the decomposition of $S(\mathfrak{g})$ with respect to the adjoint action. Every element $x$ in $\mathfrak{g}$ defines a first order differential operator $D_{x}$ on $S\left(\mathfrak{g}_{\mathbb{C}}\right)$ by

$$
D_{x}(y)=\langle x, y\rangle
$$

where $y$ is in $\mathfrak{g}=S^{1}(\mathfrak{g})$, and $\langle x, y\rangle$ is the Killing form. Let $Z$ be the subring of invariant polynomials in $S(\mathfrak{g})$. Then

$$
Z=\mathbb{C}\left[\omega_{1}, \ldots, \omega_{n}\right]
$$

where $\omega_{i}$ are invariant polynomials of degree $d_{i}+1$, and $d_{i}$ are the exponents of $\mathfrak{g}$. We shall assume that $d_{1}=1$, so that $\omega_{1}=\Omega$ is the Casimir operator. Recall that $\Omega=\sum_{i} e_{i} e_{i}^{\prime}$, where $\left(e_{i}\right)$ is any basis of $\mathfrak{g}$, and $\left(e_{i}^{\prime}\right)$ the corresponding dual basis. The following is a reinterpretation of $[\mathrm{K}]$ :

Proposition 3.2. Let $Z^{+}$be the augmentation ideal in $Z$. Let $\omega$ be in $Z^{+}$. It defines an element $A_{\omega}$ in $\operatorname{Hom}(\mathfrak{g}, U(\mathfrak{g}))$ such that $A_{\omega}(x)=D_{x}(\omega)$. Moreover, $\omega \mapsto A_{\omega}$ defines an isomorphism between $Z^{+}$and $\operatorname{Hom}(\mathfrak{g}, S(\mathfrak{g}))$, decreasing degree by one.

Now note the following proposition:

Proposition 3.3. Let $V$ be an irreducible constituent of $W$. Then $\mathfrak{g} \cdot V \subseteq S^{3}(\mathfrak{g})$ contains a submodule isomorphic to $\mathfrak{g}$.

Proof. Let $D_{\Omega}$ be the differential operator corresponding to the Casimir element $\Omega$. Invariance of $\Omega$ implies that $D_{\Omega}$ is a homomorphism from $S^{3}(\mathfrak{g})$ to $S^{1}(\mathfrak{g})$. It suffices to show that $D_{\Omega}$ is non-trivial when restricted to the subspace $\mathfrak{g} \cdot V$.

To see this, observe that for any $p \in S^{2}(\mathfrak{g})$ and $x \in \mathfrak{g}$,

$$
D_{\Omega}(x p)=2 D_{x}(p) \text {. }
$$

Thus, given non-zero $p$, one can always find $x$ such that $D_{\Omega}(x p) \neq 0$. This proves the proposition.

Remarks: In fact, the multiplicity of $\mathfrak{g}$ in $\mathfrak{g} V$ is 1 . To see this, note that one has inclusions:

$$
\operatorname{Hom}(\mathfrak{g} V, \mathfrak{g}) \subset \operatorname{Hom}(\mathfrak{g} \otimes V, \mathfrak{g})=\operatorname{Hom}(V, \mathfrak{g} \otimes \mathfrak{g})
$$


and the latter space has dimension 1, as shown in [BJ, Lemma 4.4].

We can now finish the proof of theorem. Checking out the exponents, Prop. 3.2 implies that

$$
\operatorname{dim} \operatorname{Hom}\left(\mathfrak{g}, S^{3}(\mathfrak{g})\right)=\left\{\begin{array}{l}
1, \text { if } \mathfrak{g} \text { is exceptional; } \\
2 \text { if } \mathfrak{g} \text { has type } B_{n}, C_{n}(n \geq 2) \text { or } D_{n}(n>4) \\
3 \text { if } \mathfrak{g} \text { has type } D_{4} .
\end{array}\right.
$$

Observe that this number is equal to the number of irreducible constituents of $W$.

It follows that for exceptional Lie algebras, $\Omega \cdot \mathfrak{g}$ is the unique summand in $S^{3}(\mathfrak{g})$ isomorphic to $\mathfrak{g}$. Proposition 3.3 implies that $\mathfrak{g} \cdot V\left(\alpha+\alpha_{i}\right)$ (now $\alpha_{i}$ is unique) contains $\Omega \cdot \mathfrak{g}$ and therefore the theorem follows for exceptional Lie algebras.

The above argument can be adapted to $D_{4}$ by making use of the group of outer automorphisms $\Sigma \cong S_{3}$ of $D_{4}$. The group $\Sigma$ acts on $\operatorname{Hom}\left(\mathfrak{g}, S^{3}(\mathfrak{g})\right)$ which is isomorphic to $Z^{4}$ by Prop. 3.2. The latter space can be easily decomposed under the action of $\Sigma$. It has a line fixed by $\Sigma$, and the two dimensional complement is the irreducible representation $r$ of $\Sigma \cong S_{3}$. Clearly, the fixed line in $\operatorname{Hom}\left(\mathfrak{g}, S^{3}(\mathfrak{g})\right)$ corresponds to $\Omega \cdot \mathfrak{g}$. On the other hand, since $\Sigma$ permutes the three simple roots $\alpha_{i}$, the contribution of $\sum_{i} \mathfrak{g} \cdot V\left(\alpha+\alpha_{i}\right)$ to $\operatorname{Hom}\left(\mathfrak{g}, S^{3}(\mathfrak{g})\right)$ is $\Sigma$-invariant. Next, note that $D_{\Omega}$ induces a $\Sigma$ invariant map from $\operatorname{Hom}\left(\mathfrak{g}, S^{3}(\mathfrak{g})\right)$ to $\operatorname{Hom}\left(\mathfrak{g}, S^{1}(\mathfrak{g})\right)$. It follows that $D_{\Omega}$ annihilates $r \otimes \mathfrak{g}$. On the other hand, $D_{\Omega}$ does not annihilate $\mathfrak{g} \cdot W$. It follows that $\Omega \cdot \mathfrak{g}$ is contained in $\mathfrak{g} \cdot W$, as desired.

Finally, we are left with the classical cases: $C_{n}(n \geq 2), B_{n}(n \geq 2)$ and $D_{n}$ $(n>4)$. The argument we provide below is due to N. Wallach. Here, $W$ has 2 irreducible constituents $V_{1}$ and $V_{2}$, and we need to show that the two copies of $\mathfrak{g}$ in $\mathfrak{g} \cdot V_{1}$ and $\mathfrak{g} \cdot V_{2}$ are different. By Prop. 3.2, we know that there are 2 invariant polynomials $q_{1}$ and $q_{2}$ of degree 4 which give rise to these two copies of $\mathfrak{g}$ in $S^{3}(\mathfrak{g})$. Thus, we need to show that $q_{1}$ is not a multiple of $q_{2}$.

How can one obtain the invariant $q_{i}$ from $V_{i}$ ? Well, the representation $V_{i}$ possesses an invariant symmetric bilinear form, and thus there is an invariant polynomial $Q_{i}$ in $S^{2}\left(V_{i}\right)$. If $\pi_{i}: S^{2}(\mathfrak{g}) \longrightarrow V_{i}$ is the projection, then

$$
q_{i}(x)=Q_{i}\left(\pi_{i}\left(x^{2}\right)\right) \quad \text { for } x \in \mathfrak{g} .
$$

We do not know how to show that $q_{i}$ is non-zero from general considerations. However, for the cases at hand, this will be shown in the course of the argument below.

Another description of $q_{i}$ is as follows. If $\left\{v_{j}\right\}$ is a basis of $V_{i}$, and $Q_{i}=$ $\sum_{j \leq k} a_{j k} v_{j} v_{k}$ is an invariant quadratic form on $V_{i}$, then $q_{i}$ is the image of $Q_{i}$ under the natural map $S^{2}\left(S^{2}(\mathfrak{g})\right) \longrightarrow S^{4}(\mathfrak{g})$. In other words, $q_{i}$ is equal to 
$Q_{i}$ regarded as a quartic polynomial on $\mathfrak{g}$ (by regarding the $v_{j}$ 's as quadratic polynomials on $\mathfrak{g})$. From this second description, it is clear that if $x \in \mathfrak{g}$, then

$$
D_{x}\left(q_{i}\right)=\sum_{j \leq k} a_{j k} \cdot\left(D_{x}\left(v_{j}\right) v_{k}+v_{j} D_{x}\left(v_{k}\right)\right) \in \mathfrak{g} \cdot V_{i} .
$$

This shows that $q_{i}$ indeed gives rise to a copy of $\mathfrak{g}$ in $\mathfrak{g} \cdot V_{i}$ (as long as $q_{i}$ is non-zero).

We are now ready to finish the proof for the classical cases. Let us begin with an observation. If $V$ is the standard representation of a classical $\mathfrak{g}$, so that $V$ possesses a $\mathfrak{g}$-invariant form $\langle-,-\rangle$, then $S^{k}(V)$ and $\wedge^{k} V$ inherit a non-zero $\mathfrak{g}$-invariant form:

$$
\left\{\begin{array}{l}
\left\langle a_{1} a_{2} \ldots a_{k}, b_{1} b_{2} \ldots b_{k}\right\rangle_{S^{k}(V)}=\sum_{\sigma \in S_{k}} \prod_{i}\left\langle a_{i}, b_{\sigma(i)}\right\rangle \\
\left\langle a_{1} \wedge a_{2} \ldots \wedge a_{k}, b_{1} \wedge b_{2} \ldots \wedge b_{k}\right\rangle_{\wedge^{k} V}=\sum_{\sigma \in S_{k}} \operatorname{sign}(\sigma) \prod_{i}\left\langle a_{i}, b_{\sigma(i)}\right\rangle .
\end{array}\right.
$$

In the following, we shall use these bilinear forms on the symmetric and exterior algebra.

Now consider the orthogonal case (type $B$ or $D$ ). We realize $\mathfrak{g}$ as the Lie algebra of $n \times n$ skew-symmetric matrices, so that $\mathfrak{g} \cong \wedge^{2} \mathbb{C}^{n}$. Let us write down the invariant polynomial $q_{1}$. The map

$$
\pi_{1}^{\prime}(a \otimes b)=\frac{1}{2}(a b+b a), \quad a, b \in \mathfrak{g}
$$

defines an equivariant map from $S^{2}(\mathfrak{g})$ onto the space $S^{2}\left(\mathbb{C}^{n}\right)$ of $n \times n$ symmetric matrices. Since $S^{2}\left(\mathbb{C}^{n}\right) \cong \mathbb{C} \oplus V_{1}$, the projection $\pi_{1}$ is given by:

$$
\pi_{1}(a \otimes b)=\pi_{1}^{\prime}(a \otimes b)-\frac{1}{n} \operatorname{Tr}\left(\pi_{1}^{\prime}(a \otimes b)\right) \cdot I_{n}
$$

where $I_{n}$ is the identity matrix and $\operatorname{Tr}$ is the trace map. Since the degree 2 invariant on $V_{1}$ is simply the map $X \mapsto \operatorname{Tr}\left(X^{2}\right)$, we get:

$$
q_{1}(a)=\operatorname{Tr}\left(\left(\pi_{1}(a \otimes a)\right)^{2}\right) .
$$

In particular, if $a_{0}=e_{1} \wedge e_{2} \in \wedge^{2} \mathbb{C}^{n}$, a simple computation shows that

$$
q_{1}\left(a_{0}\right)=\frac{2}{n} \cdot(n-2) \neq 0
$$

On the other hand, the representation $V_{2}$ is isomorphic to $\wedge^{4} \mathbb{C}^{n}$ (this is irreducible if $n=5,7$ or $n>8$ ), and the projection $\pi_{2}: S^{2}(\mathfrak{g}) \longrightarrow \wedge^{4} \mathbb{C}^{n}$ is given by:

$$
\pi_{2}(a \otimes b)=a \wedge b \quad \text { for } a, b \in \mathfrak{g} .
$$

Since $\pi_{2}\left(a_{0} \otimes a_{0}\right)=0$, we deduce that

$$
q_{2}\left(a_{0}\right)=Q_{2}\left(\pi_{2}\left(a_{0} \otimes a_{0}\right)\right)=0 .
$$

Now to see that $q_{2}$ is non-zero, note that for $x \in \mathfrak{g}=\wedge^{2} \mathbb{C}^{n}$, we have

$$
q_{2}(x)=\langle x \wedge x, x \wedge x\rangle_{\wedge^{4} \mathbb{C}^{n}},
$$


and a short computation gives:

$$
q_{2}\left(e_{1} \wedge e_{2}+e_{3} \wedge e_{4}\right)=1
$$

We thus conclude that $q_{1}$ and $q_{2}$ are non-zero and linearly independent, as desired.

Finally, we come to the case $C_{n}(n \geq 2)$, which is somewhat more complicated. Let $\left\{e_{1}, e_{2}, . ., e_{n}, e_{-1}, e_{-2}, \ldots, e_{-n}\right\}$ be the standard basis for $\mathbb{C}^{2 n}$, equipped with the skew-symmetric form $\omega$ defined by $\omega\left(e_{i}, e_{-j}\right)=\delta_{i j}$ and $\omega\left(e_{i}, e_{j}\right)=\omega\left(e_{-i}, e_{-j}\right)$ $=0$. We identify $\mathfrak{g}$ with the space $S^{2}\left(\mathbb{C}^{2 n}\right)$ of $2 n \times 2 n$ symmetric matrices.

Let $V_{1}=V\left(\alpha+\alpha_{1}\right)$ and $V_{2}=V\left(\frac{1}{2}\left(\alpha+\alpha_{1}\right)\right)$. Note that $\wedge^{2} \mathbb{C}^{2 n}=\mathbb{C} \omega \oplus V_{2}$ where

$$
\omega=\sum_{i=1}^{n} e_{i} \wedge e_{-i}
$$

Thus, we have

$$
S^{2}\left(\wedge^{2} \mathbb{C}^{2 n}\right) \cong \mathbb{C} \omega^{2} \oplus \omega \cdot V_{2} \oplus S^{2}\left(V_{2}\right) .
$$

In particular, $S^{2}\left(\wedge^{2} \mathbb{C}^{2 n}\right)$ contains $V_{1}$ with multiplicity one but does not contain $V(2 \alpha)$.

Now consider the equivariant map

$$
\phi: S^{2}(\mathfrak{g})=S^{2}\left(S^{2} \mathbb{C}^{2 n}\right) \longrightarrow S^{2}\left(\wedge^{2} \mathbb{C}^{2 n}\right)
$$

given by

$$
(a \cdot b) \otimes(c \cdot d) \mapsto(a \wedge c) \cdot(b \wedge d)+(a \wedge d) \cdot(b \wedge c)
$$

We claim that the image of $\phi$ is isomorphic to $\mathbb{C} \oplus V_{1} \oplus V_{2}$. Indeed, $V_{1}$ is in the image of $\phi$, since

$$
\phi\left(\left(e_{1} \cdot e_{2}\right) \otimes\left(e_{1} \cdot e_{2}\right)\right)=-\left(e_{1} \wedge e_{2}\right)^{2}
$$

is a highest weight vector of weight $\alpha+\alpha_{1}$. On the other hand, if

$$
\pi: S^{2}\left(\wedge^{2} \mathbb{C}^{2 n}\right) \longrightarrow \wedge^{2} \mathbb{C}^{2 n}
$$

is given by:

$$
(a \wedge b) \cdot(c \wedge d) \mapsto \omega(a, b) \cdot(c \wedge d)+\omega(c, d) \cdot(a \wedge b)
$$

then one easily checks that $\pi \circ \phi$ is a surjective equivariant map from $S^{2}(\mathfrak{g})$ to $\mathbb{C} \omega \oplus V_{2}$.

Now let

$$
a=\sum_{i=1}^{n} e_{i}^{2}+\sum_{i=1}^{n} e_{-i}^{2} \in S^{2}\left(\mathbb{C}^{2 n}\right)=\mathfrak{g} .
$$

Then a short computation shows that

$$
\pi \circ \phi\left(a^{2}\right)=8 \omega
$$


Thus, if $\pi_{2}: S^{2}(\mathfrak{g}) \rightarrow V_{2}$ is the projection map, then $\pi_{2}\left(a^{2}\right)=0$ so that $q_{2}(a)=0$. It is not difficult to check that $q_{2} \neq 0$ by evaluating at the element $e_{1} e_{-1}$ say. Indeed, one has:

$$
\pi \circ \phi\left(\left(e_{1} e_{-1}\right)^{2}\right)=-2 e_{1} \wedge e_{-1} \in \wedge^{2} \mathbb{C}^{2 n}
$$

and so

$$
\pi_{2}\left(\left(e_{1} e_{-1}\right)^{2}\right)=-2 e_{1} \wedge e_{-1}+\frac{2}{n} \omega .
$$

Then a short computation gives:

$$
q_{2}\left(e_{1} e_{-1}\right)=\frac{4}{n} \cdot(n-1) \neq 0 .
$$

It remains to show that $q_{1}(a) \neq 0$. Let $q \in S^{2}(\mathfrak{g})$ correspond to the $\mathfrak{g}$-invariant form $\langle-,-\rangle_{S^{2}\left(\mathbb{C}^{2 n}\right)}$, so that $q$ is a multiple of the Killing form. We have:

$$
q=\sum_{i=1}^{n} e_{i}^{2} \cdot e_{-i}^{2}+2 \sum_{i<j}\left(e_{i} e_{j}\right) \cdot\left(e_{-i} e_{-j}\right)-\sum_{i=1}^{n}\left(e_{i} e_{-i}\right)^{2}-\sum_{i \neq j}\left(e_{i} e_{-j}\right)\left(e_{-i} e_{j}\right) .
$$

One checks that

$$
\pi \circ \phi(q)=(4 n+2) \omega
$$

and thus the element

$$
\pi_{1}\left(a^{2}\right)=\phi\left(a^{2}\right)-\frac{4}{2 n+1} \phi(q)
$$

lies in $V_{1} \subset$ Image $(\phi)$. Now if $\langle-,-\rangle$ denotes the standard invariant symmetric bilinear form on $S^{2}\left(\wedge^{2} \mathbb{C}^{2 n}\right)$, then a somewhat messy computation, best suppressed here, gives:

$$
q_{1}(a)=\left\langle\pi_{1}\left(a^{2}\right), \pi_{1}\left(a^{2}\right)\right\rangle \neq 0 .
$$

This shows that $q_{1}$ and $q_{2}$ are linearly independent, as desired.

Theorem 3.1 is proved completely.

\section{Acknowledgments}

It is a pleasure to thank Nolan Wallach for his interest in this work and for his help in the proof of the classical case of the main theorem. We also thank A. Joseph and A. Braverman for bringing their paper [BJ] to our attention.

The first author is partially supported by the AMS Centennial fellowship and NSF grant DMS-0352682. The second author is partially supported by NSF grant DMS-0138604. 


\section{References}

[BJ] A. Braverman and A. Joseph, The minimal realization from deformation theory, Journal of Algebra 205 (1998), 13-36.

[G] D. Garfinkle, A new construction of the Joseph ideal, PhD thesis, MIT.

$[\mathrm{J}]$ A. Joseph, The minimal orbit in a simple Lie algebra and its associated maximal ideal, Ann. Sci. Ecole Norm Sup. 9 (1976), 1-29.

[K] B. Kostant, Lie group representations on polynomial rings, American J. Math. 85 (1963), 327-404.

[S] G. Savin, A letter to David Vogan, January 16, 2002.

Department of Mathematics, University of California-San Diego, La Jolla, CA 92093, USA

E-mail address: wgan@math.ucsd.edu

Department of Mathematics, University of Utah, Salt Lake City, UT 84112, USA

E-mail address: savin@math.utah.edu 